\title{
EDITORIAL
}

cell biology

\section{Colouring in cells}

\section{This year's Nobel Prizes mark the most significant tech- nological advance in cell biology, GFP et al., as well as two discoveries in virology with major health implications.}

In the last decade a number of technological breakthroughs have completely changed the type of data we can extract from cells. Selective loss of gene function in organisms and cells was celebrated with last year's Nobel prize for Medicine, which recognized mouse knockout technology, and the year before, RNAi made an uncharacteristically early appearance in Stockholm. Future prizes are likely to revisit the two prizes Fred Sanger received many years ago for DNA and protein sequencing to mark genome sequencing and proteomics. This year the Nobel Prize in Chemistry celebrates what is undoubtedly the most important current tool for the cell biologist: green fluorescent protein (GFP) and its rainbow of derivatives.

Osamu Shimomura, one of the three awardees, first isolated GFP from the jellyfish Aequorea victoria in 1962, at the Woods Hole Marine Biological Laboratory and there, thirty years later, Douglas Prasher cloned the GFP gene. Prasher has been considered equally deserving of the prize by the other awardees. However, the experimental potential was noted only fourteen years ago by Nobel Laureate Martin Chalfie of Columbia University (NY), who expressed it in Escherichia coli and in his favoured model organism, the transparent roundworm Caenorhabditis elegans, as a marker for $\beta$-tubulin gene expression (see also page 1234 of this issue). In the same year Wang and Hazelrigg first used GFP fused to proteins in Drosophila melanogaster. The great advantage of GFP-tagging over other tags and antibody-based approaches is of course that almost any protein can be tagged - usually without interfering with its function - and thus visualized with fluorescence microscopy in living cells, organs or indeed whole organisms (even opaque ones these days). The technique affords a minimally invasive visual tool to track a specific biomolecule in real time in its native environment, and at physiological expression levels.

Roger Tsien (University of California, San Diego), who shares the Nobel Prize with Shimomura and Chalfie, has spearheaded research since 1994 that has dramatically increased the potential of GFP by optimizing its fluorescence characteristics and increasing the palette of fluorophores through mutagenesis and cloning of other fluorescent proteins. These breakthroughs have been mirrored by marked advances in light microcopy, such as confocal microscopy and total internal reflection fluorescence (TIRF) microscopy.

As a result, a number of techniques with bewildering four letter acronyms (starting with F) have evolved. These include fluorescence (or Förster) resonance energy transfer (FRET), which measures protein interactions and allows the design of biosensors that can, for example, monitor the activity of a protein kinase; fluorescence recovery after photobleaching (FRAP), which measures protein mobility and dynamics by monitoring the reappearance of a biomolecule in a photobleached area, and the related fluorescence loss in photobleaching (FLIP); fluorescence lifetime imaging microscopy (FLIM), which is used to measure biomolecular concentrations and interactions. Notably,
Shimomura actually described FRET between blue luminescent jellyfish aequorin and GFP in 1974.

Experiments unimaginable just a decade ago are now a staple at NCB: this issue is typical with eleven of the thirteen papers presenting GFP-based data. By facilitating the quantitative study of biomolecules in the three-dimensional context of the cell or organ, and by allowing measurement of their dynamics in real time, fluorescent tags have allowed us to enter a completely new level of biological experimentation. It is fair to say that through GFP-based techniques, cell biology has come of age as a quantitative life science (with real-Live cells).

One recent application of GFP was to document the assembly of individual virions of human immunodeficiency virus (HIV) in real time and in live cells. This study forms part of a major research effort whose origin was marked by this year's Nobel Prize for Physiology or Medicine, awarded to Françoise Barré-Sinoussi and Luc Montagnier for their discovery of HIV-1 in 1983. The award had been expected for many years, but the delay seems wise as it has allowed the rather unsightly dust stirred up at the time about credit for the discovery to settle. The award for the discovery of HIV was bound to be hotly debated because of the fundamental contributions Robert Gallo (University of Maryland, Baltimore) made to HIV/AIDS research right from the beginning by isolating HIV-1 from many patients in 1984, and confirming its causative role in the development of AIDS (alongside Montagnier, Jay Levy, Robin Weiss, Max Essex and William Haseltine). The Nobel committee evidently noted the detailed forensic investigations into the history of the discovery of HIV, published in the wake of conflicting claims from the two main groups, and made a clear statement by awarding half of this year's prize to Harald zur Hausen of the German Cancer Research Centre (Heidelberg) for his discovery of human papilloma viruses (HPVs) that cause cervical cancer. As with the discovery of HIV, the recognition that HPVs are the causative agents of over $70 \%$ of cervical cancers has significant health implications and the award is timely as immunization is now widely available, although still faced with a worrying amount of opposition in many countries.

Earlier this year, the Lasker Basic Medical Research Award to David Baulcombe (University of Cambridge), Victor Ambros (University of Massachusetts Medical School, Worcester) and Gary Ruvkun (Massachusetts General Hospital, Boston) carried the citation 'For discoveries that revealed an unanticipated world of tiny RNAs that regulate gene function in plants and animals.' Remarkably, the miRNA work of Ambros and Ruvkun started with lin-4 and lin-14, genes identified by Chalfie and colleagues. As with the implication of HPV16 and 18 in cervical cancer, which went against the prevailing dogma and took more than ten years to be accepted, the discovery of small inhibitory RNAs was totally unexpected and took many years to unravel, partly because of its 'dogma shifting' attributes. The tenacity, creativity and, in Baulcombe's words, "left hemisphere thinking", alone richly deserves the prize, besides the significant impact these findings have had in providing the major research tool noted above and in uncovering a whole new dimension of cellular regulation. It is satisfying that the Lasker Prize, normally seen as presage to the Nobel Prize, revisited the topic two years after the Nobel Prize awarded to Andrew Fire and Craig Mello to recognize three arguably equally deserving small RNA pioneers. 